Bundesgesundheitsbl 2020 $63: 137-144$ https://doi.org/10.1007/s00103-019-03078-7 Online publiziert: 9 . Januar 2020

c) Der/die Autor(en) 2019

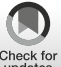

Hajo Zeeb ${ }^{1,2,3}$ für Leibniz-WissenschaftsCampus Digital Public Health Bremen . Iris Pigeot ${ }^{1,2,4}$ für Leibniz-WissenschaftsCampus Digital Public Health Bremen . Benjamin Schüz ${ }^{1,5}$ für Leibniz-WissenschaftsCampus Digital Public Health Bremen 'Leibniz WissenschaftsCampus Digital Public Health Bremen, Bremen, Deutschland ${ }^{2}$ Leibniz-Institut für Präventionsforschung und Epidemiologie - BIPS, Bremen, Deutschland ${ }^{3}$ Fachbereich Human- und Gesundheitswissenschaften, Universität Bremen, Bremen, Deutschland ${ }^{4}$ Fachbereich Mathematik und Informatik, Universität Bremen, Bremen, Deutschland

${ }^{5}$ Fachbereich Human- und Gesundheitswissenschaften, Institut für Public Health und Pflegeforschung, Universität Bremen, Bremen, Deutschland

\title{
Digital Public Health - ein Überblick
}

schende und Akteure im Bereich Public Health stellen müssen, auch um selber eine gestaltende und führende statt einer passiven Rolle in der hochdynamischen Entwicklung einzunehmen.

Noch harren allerdings selbst definitorische Fragen einer Klärung: Begrifflichkeiten wie E-Health und mobile (M-) Health, E-Public-Health oder eben Digital Public Health werden im Kontext der Digitalisierung von Public Health gebraucht und Digital Health, Health 2.0, Cybermedizin, digitale Medizin und $\mathrm{Te}-$ lemedizin sind ebenfalls in begrifflicher Nähe angesiedelt. So versteht sich z. B. das Journal of Medical Internet Research als führende wissenschaftliche Zeitschrift für digitale Medizin, Gesundheit und Gesundheitsversorgung im Internetzeitalter. Ein im Juli 2019 dort eingereichter Beitrag zu Konzepten von E-Health [7] bestätigt auf der Basis einer umfassenden Literaturrecherche den fehlenden Konsens bei der Definition von E-Health als vermutlich historisch erstem Begriff in diesem Feld. Ein Review aus dem Jahr 2005 fand schon 51 verschiedene Definitionen [8] und neuere Arbeiten betonen, dass sich das Feld aufgrund seiner großen Dynamik möglicherweise einer klaren Definition entzieht [9]. Die Autoren der aktuellen Übersicht [7] leiten aus den unterschiedlichen Aspekten eine gemeinsame Definition ab, die E-Health als Bereitstellung nutzerzentrierter Gesundheitsdienstleistungen durch Informations- und Kommunikationstechno- logien, und zwar vornehmlich das Internet, beschreibt.

Der Fokus auf Internetanbindung findet sich auch im Papier zu „Digital-first public health" von Public Health England [10] - hier wird "digital“ als „applying the culture, practices, processes \& technologies of the internet era to respond to people's raised expectations" verstanden.

Der Begriff „M-Health“ ist hingegen spezifischer auf eine bestimmte Technologie, nämlich mobile Smartphones und Sensoren, in ihrer Bedeutung für Gesundheit ausgerichtet und damit insgesamt klarer definiert, auch wenn hier verschiedene Technologien zum Einsatz kommen [11]. Diese auf die Technologie ausgerichtete Definition führt jedoch dazu, dass eine Zuordnung zu klinischer Medizin und Versorgung oder Public Health nicht klar ist. M-Health wird dabei als ein der E-Health untergeordneter Bereich angesehen. Kabellose und mobile Technologien und ihr Einsatz in der Verbesserung der gesundheitsbezogenen Forschung, der Versorgung und letztendlich des Gesundheitsstatus stehen im Mittelpunkt [9].

"Digital Health“ ist als umfassender Begriff für die Anwendung von Informations- und Kommunikationstechnologien im Zusammenhang mit Versorgung und Bevölkerungsgesundheit anzusehen [12], der sich kaum von E-Health oder Health 2.0 unterscheidet. Kooperation und Interaktion bei der Nutzung digitaler Technologien sind oftmals ein esein Zukunftsthema ist, dem sich For- 
senzieller Bestandteil der definitorischen Beschreibung.

"Digital Public Health" wiederum fokussiert das Entwicklungs-, Anwendungs- und Erkenntnisinteresse auf Public Health und damit auf Prävention, Gesundheitsförderung sowie die entsprechenden Grundwissenschaften wie Epidemiologie. Primär klinische und individuell auf Patientinnen und Patienten bezogene Aspekte stehen nicht im Vordergrund, anders als z. B. bei der Telemedizin mit der konkreten Anwendung im individuellen Behandlungs- und Versorgungskontext. Festzuhalten ist allerdings, dass sich der Begriff Digital Public Health bisher nicht gegenüber anderen wie E-Health und M-Health durchgesetzt hat und dies auch angesichts der bisherigen Begriffsvielfalt und Dynamik kaum zu erwarten ist. Wo jedoch in Hinsicht auf Digitalisierung und Gesundheit der Fokus auf Bevölkerungen, Prävention und Gesundheitsförderung samt einer bewussten Auseinandersetzung gesundheitlicher Ungleichheiten gelegt wird, kann Digital Public Health eine klarere Einordnung bieten als einige andere Begriffe in diesem Feld.

In der Folge diskutiert dieser Beitrag im Sinne einer orientierenden Bestandsaufnahme wichtige Aspekte und Herausforderungen von Digital Public Health. Hervorgehoben werden besondere Ansprüche sowie Rahmenbedingungen des Datenschutzes bei der digitalen Kommunikation und Interaktion bezüglich Gesundheitsdaten und die Thematik der sogenannten digitalen Spaltung (engl. Digital Divide) mit ihren verschiedenen Facetten.

\section{Digital Public Health: Versuch einer Bestandsaufnahme}

Aus der Kombination der Implikationen der Begriffe „digital“ und „Public Health“e ergibt sich aus den oben angedeuteten Hintergründen ein herausforderndes Spannungsfeld, in dem wissenschaftlich fundierte Erkenntnisse aus der Public Health ebendiesen gestiegenen Erwartungen aus der Internetära gegenüberstehen. Für Digital Public Health bedeutet dies insbesondere, dass dem ubiquitären technologischen Fortschritt und den sich beständig erweiternden Möglichkeiten zur Gestaltung, Dissemination und Kommunikation gesundheitlicher Themen ein Vorgehen gegenübergestellt werden sollte, in dem es primär um die Erreichung von Public-Health-Zielen wie die allgemeine Verbesserung der Gesundheit und die Verringerung von gesundheitlicher Ungleichheit geht.

Während es aber in manchen Überlegungen zu Digital Public Health darum geht, Public Health im Angesicht der technologischen Entwicklung neu zu definieren und zu konzeptualisieren [10], liegt aus unserer Sicht die zentrale Herausforderung darin, digitale Entwicklung und Technologien in Konzepte von Public Health zu integrieren und so zur Erreichung von Public-Health-Zielen zu nutzen.

Dabei ist es besonders wichtig, neben den Potenzialen digitaler Technologien in Public Health [13] auch auf die Implikationen für gesundheitliche Ungleichheit einzugehen. Einerseits besteht durch die Skalierbarkeit von digitalen Interventionen und minimalen Kosten pro Nutzer bzw. Nutzerin zumindest theoretisch die Möglichkeit, große Teile der Bevölkerung für digitale Angebote zu erreichen. Andererseits zeigt sich durch die differenzielle Nutzung und Verbreitung von digitalen Gesundheitsangeboten, dass hauptsächlich bereits gesundheitlich privilegierte Gruppen von solchen Angeboten profitieren und so gesundheitliche Ungleichheiten möglicherweise noch vergrößert werden [14].

Bisherige Übersichtsarbeiten im deutschsprachigen (z. B. [15]) und internationalen Kontext (z. B. [7]) orientieren sich an Begriffen wie E-Health und M-Health. Während diese Definitionen und Kategorisierungen hilfreich sind, um unterschiedliche Technologien $\mathrm{zu}$ beschreiben und einen Überblick über das Feld zu bekommen, sind sie noch nicht an den Bedarfen von Public Health und dem Prozess der Entwicklung und Evaluierung von Maßnahmen zur Erreichung von Public-Health-Zielen (also grundlegenden Prozessen evidenzbasierter Public Health; [16]) orientiert.

Grundsätzlich mangelt es in diesem Kontext bisher an gut geplanten und durchgeführten Evaluationsstudien und angemessenen Evaluationsparadigmen. Dies wird in mehreren Überblicksarbeiten zu digitalen Gesundheitstechnologien deutlich [17], und zwar sowohl im Allgemeinen [18] als auch im Kontext spezifischer Interventionsparadigmen, wie z. B. Just-In-Time-AdaptiveInterventionen (also Interventionen, die individualisierte Inhalte in genau dem Moment mit größter erwarteter Wirkung vermitteln [19]). Ein Problem bei der Entwicklung solcher angepassten Evaluationsparadigmen kann auch darin begründet liegen, dass digitale Gesundheitstechnologien bislang oft nach Technologie oder Zielverhalten, nicht aber nach zentralen Funktionen in Public Health diskutiert wurden.

\section{Digital Public Health: Kategorien nach Funktionen}

Eine Kategorisierung von digitalen Gesundheitstechnologien nach zentralen Public-Health-Funktionen impliziert, dass Technologien nicht nach Plattform und Software getrennt diskutiert werden (z.B. Apps für Mobiltelefone getrennt von internetbasierten Angeboten, die hauptsächlich am PC durchgeführt werden), sondern dass die Funktionen der Technologie für die Erreichung von Gesundheitszielen als primäre Systematik der Beschreibung dienen.

Eine solche Kategorisierung ist nichts grundsätzlich Neues: Im Vereinigten Königreich hat beispielsweise das National Institute for Health and Care Excellence (NICE) 2019 eine Systematik von digitalen Technologien im Gesundheitsbereich vorgestellt [20], die als Grundlage eines Überblicks über die Angebote im Feld Digital Public Health dienen kann. Diese Klassifikation orientiert sich an den Funktionen von digitalen Angeboten im Gesundheitsbereich im Hinblick auf Nähe und Interaktionstiefe mit den Nutzern und Nutzerinnen und ordnet diesen Funktionen verschiedene Anforderungen an Evidenzgüte zu (• Abb. 1).

Auf der ersten Ebene finden sich entsprechend digitale Technologien, die prinzipiell der Verbesserung der Organisation des Gesundheitswesens dienen, sich aber eher nicht direkt auf die Gesundheit oder Determinanten von 
Gesundheit von Einzelpersonen oder Gruppen auswirken. Auf der zweiten Ebene finden sich Technologien, die direkt mit Endnutzern aus der Bevölkerung interagieren, sich aber auf die Information, einfache Monitoringaufgaben und Kommunikation beziehen. Dies könnten beispielsweise Apps sein, die über einen gesunden Lebensstil informieren, elektronische Tagebücher für Symptome oder körperliche Aktivität oder digitale Plattformen zur Kommunikation zwischen Personen und Akteuren aus dem Gesundheitswesen. Natürlich bieten solche Technologien auch die Möglichkeit, Inhalte $\mathrm{zu}$ vermitteln, die sich direkt oder indirekt auf Gesundheit auswirken - allerdings ist in diesem Fall die Technologie hauptsächlich Transportoder Kommunikationsmedium, nicht unbedingt wirksamer Bestandteil einer Intervention oder Therapie.

Auf der in zwei Subkategorien aufgeteilten dritten Ebene finden sich zum einen Technologien, die zur Behandlung und Prävention von Krankheiten herangezogen werden können und parallel zu einer konventionellen Therapie laufen, zum anderen Technologien, die direkt in den Therapieablauf eingreifen und Teil des Therapieprozesses werden. Beispiele für die erste Subkategorie (Ebene 3a) sind Apps, die z. B. Lebensstiländerungen wie Rauchstopps aktiv bewirken und unterstützen sollen. Beispiele für die zweite Subkategorie (Ebene 3b) sind Sensoren, die automatisch Informationen über den momentanen Gesundheitszustand (z. B. Herzschlagfrequenz oder Blutdruck) an ein Entscheidungssystem übermitteln, um so die Therapieentscheidungen für diese Person zu beeinflussen.

Das NICE-Framework von digitalen Technologien im Gesundheitsbereich orientiert sich also an den Zwecken digitaler Technologie für bestimmte Gesundheitsziele. Während der Zweck dieses Rahmenkonzepts vor allem darin besteht, die Anforderungen für Evidenzgüte in den unterschiedlichen Kategorien zu begründen, ist eine solche funktionsbezogene Herangehensweise auch für Forschungszwecke sehr nützlich. Wir möchten daher im Folgenden eine Kategorisierung von digitalen Technologien in Public Health vornehmen, die sich an

Bundesgesundheitsbl 2020 -63:137-144 https://doi.org/10.1007/s00103-019-03078-7

(c) Der/die Autor(en) 2019

H. Zeeb für Leibniz-WissenschaftsCampus Digital Public Health Bremen · I. Pigeot für LeibnizWissenschaftsCampus Digital Public Health Bremen • B. Schüz für LeibnizWissenschaftsCampus Digital Public Health Bremen

\section{Digital Public Health - ein Überblick}

\section{Zusammenfassung}

Die rasante Entwicklung neuer digitaler Technologien hat nicht nur die medizinische Praxis verändert, sondern bietet auch große Chancen und Herausforderungen für Public Health, insbesondere in Gesundheitsförderung und Prävention.

Gleichzeitig ist dieses neue Feld auch gekennzeichnet durch begriffliche und konzeptuelle Unschärfen, einen bemerkenswerten Mangel an qualitativ hochwertiger Evidenz sowie eine fehlende Diskussion von unerwünschten Effekten und Begleiterscheinungen. Eine weitere Herausforderung liegt darin, dass die Entwicklung von Technologien aktuell durch den technologischen Fortschritt und weniger durch evidenzbasierte und evidenzorientierte Forschung vorangetrieben wird.

In diesem Überblicksartikel wird das Feld

„Digital Public Health“ konzeptuell beschrie- ben und anhand von grundlegenden PublicHealth-Funktionen als Anforderungsprofil definiert. Wir diskutieren einige Beispiele, wie digitale Technologien aktuell zur Erfüllung von Public-Health-Aufgaben genutzt werden, und schlagen eine bedarfsorientierte Entwicklung von digitalen Gesundheitstechnologien vor.

Wir gehen außerdem auf spezifische Herausforderungen, insbesondere sozioökonomische Unterschiede in der Nutzung und in den Effekten von digitalen Gesundheitstechnologien, sowie Datenschutz- und ethische Aspekte ein.

\section{Schlüsselwörter}

Datenschutz · Digitalisierung · E-Health .

M-Health · Ungleichheit

\section{Digital public health—an overview}

\section{Abstract}

The rapid development and proliferation of digital health technologies have not only changed the medical professions, but offer great potential for public health, particularly in health promotion and disease prevention. At the same time, this emerging field is also characterized by conceptual and terminological fuzziness, a marked lack of high-quality evidence, and an absence of an honest discussion of unintended consequences and side effects. Further challenges for digital public health lie in the fact that the development of new health technologies is mainly driven by technological progress and less by evidence-based needs and research in public health.

In this overview paper, we aim at conceptually denoting the field of digital public health, using principal public health functions as guiding principles. We discuss some current applications of digital health technologies in fulfilling public health functions and propose a needs-based development of digital health technologies.

We will further address specific challenges to digital public health, in particular socioeconomic differences in the usage of and profiting from digital health technologies, data protection and privacy issues, as well as ethical issues.

\section{Keywords}

Data protection - Digitization - E-Health . M-Health · Inequity der Funktion von Technologie und nicht an der Technologie selbst orientiert.

\section{Zentrale Funktionen von Digital Public Health}

Die Weltgesundheitsorganisation (WHO; [21]) schlägt zehn zentrale Funktionen von Public Health („essential health operations") vor, deren Verwendung sich auch im deutschen Kontext anbietet ([22]; • Tab. 1). In allen diesen Bereichen lassen sich digitale Technologien verorten und anwenden und auf ihr Potenzial und ihre Risiken für Public Health hin untersuchen.

Einige dieser Bereiche sollen im Folgenden exemplarisch aufgegriffen und 


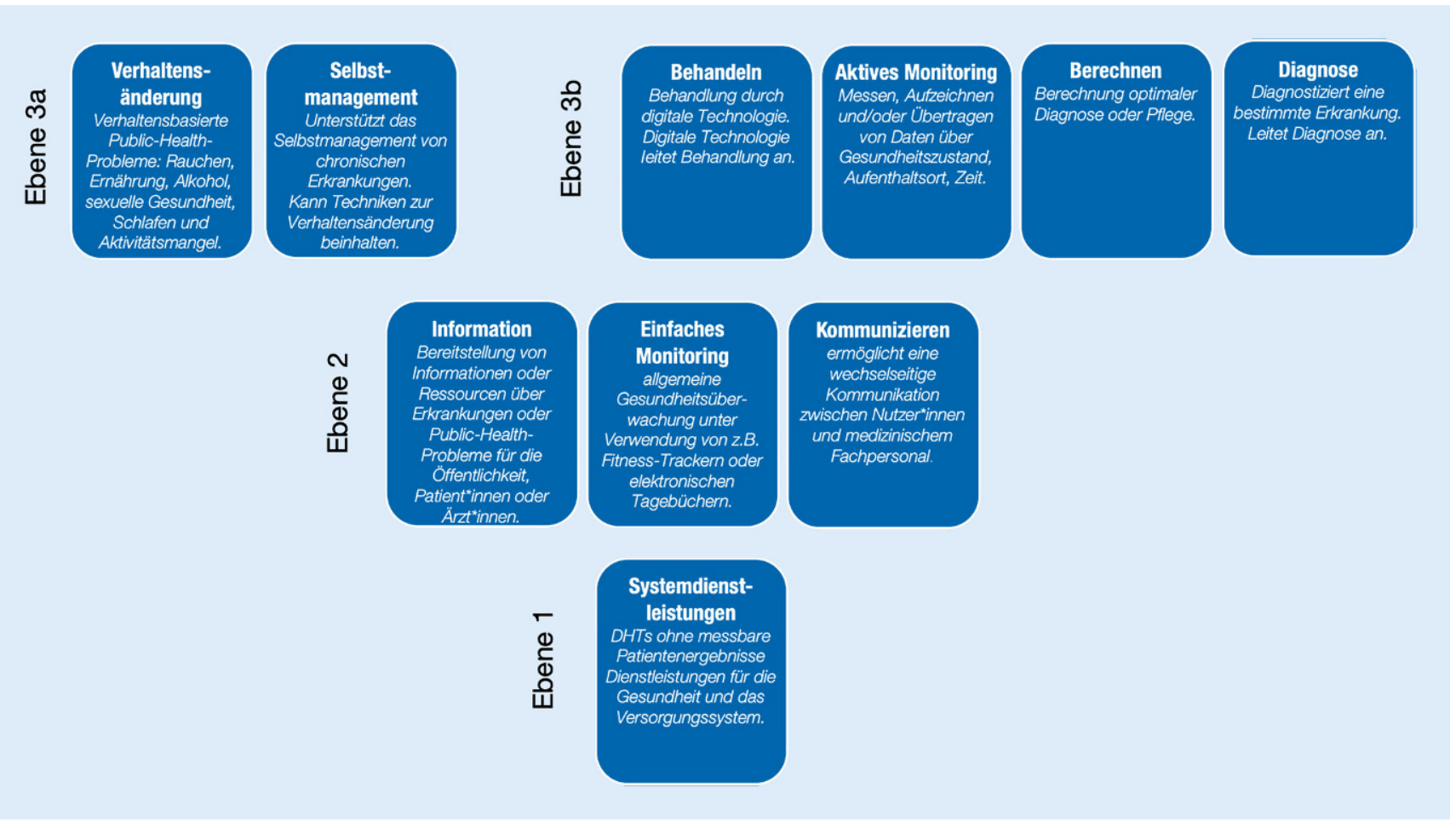

Abb. 1 A Evidenzanforderungen an digitale Gesundheitstechnologien (Digital Health Technologies [DHTs]) in Public Health nach der Tiefe der Interaktion mit Nutzer*innen (Ebene 1: geringste Interaktion; Ebene 3b: intensivste Interaktion). (Nach: National Institute for Health and Care Excellence [20]; eigene Darstellung und Übersetzung)

aktuelle Entwicklungen in der Forschung diskutiert werden. Allerdings liegen nicht in allen Bereichen gut evaluierte und evidenzbasierte Lösungen vor, daher beschränken wir uns auf einige wenige, subjektiv ausgewählte Beispiele und verweisen für eine tiefer greifende Diskussion auf die weiteren Beiträge in diesem Themenheft.

\section{Überwachung von Krankheiten und Beurteilung der Gesundheit der Bevölkerung}

Hier sind vor allem Arbeiten auf dem Gebiet der digitalen Epidemiologie [23] von Interesse. In diesem Bereich werden vor allem Daten, die durch digitale Anwendungen, wie z.B. Apps oder Fitnesstracker, auf mobilen Endgeräten generiert werden, gesammelt und zum Monitoring von Krankheiten verwendet. Dabei werden zwei grundlegend unterschiedliche Zugänge zu den Daten diskutiert [24] ein passiver Zugang, bei dem ohne $\mathrm{Zu}$ stimmung der Teilnehmenden anonymisiert z. B. Suchanfragen, Twitter-Einträge oder Ähnliches analysiert werden, oder ein partizipativer Zugang, in dem Freiwillige ihre Daten in ein bereitgestelltes Überwachungssystem eintragen und so zum Monitoring beitragen. Beispiele dafür finden sich z. B. im europäischen Influenzanet-Konsortium, in dem während der wiederkehrenden Influenzawellen Daten von Freiwilligen gesammelt werden und so zum Monitoring von Erkrankungen beitragen [25].

\section{Bestimmung vorrangiger Gesundheitsprobleme und Gesundheitsgefahren}

Hier lassen sich durch den Einsatz digitaler Technologien beispielsweise alternative Prognosemethoden für Erkrankungswellen realisieren. Eine viel beachtete Arbeit [26] hat durch die Untersuchung von zeitlichen und örtlichen Kumulationen von Suchbegriffen in der Suchmaschine Google Verbindungen zu real auftretenden Influenzawellen hergestellt, die teilweise präziser und schneller als die Vorhersagen der US-Bundesbehörde Centers for Disease Control and Prevention (CDC) waren. Allerdings können an diesem Beispiel auch die Schwächen solch indirekter digitaler epidemiologischer Forschung aufgezeigt werden [27]: Durch die gleichzeitige Berichterstattung und Diskussion von Symptomen in sozialen Medien können Internetsuchen nach relevanten Begriffen, die durch die Lektüre von Nachrichten angeregt wurden, nicht von denen unterschieden werden, die aufgrund von Symptomen erfolgen. Obwohl Algorithmen zur Erkennung solcher Muster ständig angepasst werden, ist hier weiterer Forschungsbedarf gegeben. Zudem sind mit diesen Ansätzen bisher vornehmlich bestimmte Arten von Gesundheitsproblemen - insbesondere aus dem Bereich der übertragbaren Erkrankungen - erforschbar, wobei die Ausweitung auf Veränderungen bei Risikofaktoren für chronische Erkrankungen denkbar ist (beispielsweise zur Analyse der Nutzung neuer Formen des Rauchens). 
Tab. 1 Zehn zentrale Funktionen von Public Health nach: WHO [21]. (Übersetzung nach: Nationale Akademie der Wissenschaften Leopoldina [22])

1 Überwachung von Krankheiten und Beurteilung der Gesundheit der Bevölkerung

2 Bestimmung vorrangiger Gesundheitsprobleme und Gesundheitsgefahren in der Gemeinschaft

3 Vorsorge und Planung für Notfälle im Bereich der öffentlichen Gesundheit

4 Gesundheitsschutzmaßnahmen (Umwelt-, Arbeits- und Nahrungsmittelsicherheit)

5 Krankheitsprävention

$6 \quad$ Gesundheitsförderung

7 Gewährleistung von kompetentem Personal in den Bereichen der Gesundheitsversorgung

8 Zentrale Führung, Finanzierung und Qualitätssicherung im Bereich der öffentlichen Gesundheit

9 Kernkommunikation in Bezug auf öffentliche Gesundheit

10 Gesundheitsforschung

\section{Vorsorge und Planung für Notfälle im Bereich der öffentlichen Gesundheit}

Insbesondere in diesem Bereich spielen digitale Medien, hier vor allem soziale Medien, eine entscheidende Rolle [28]. Sowohl formale Programme von internationalen Organisationen wie dem Internationalen Komitee des Roten Kreuzes (z.B. [29]) als auch informelle Informationskanäle wie WhatsApp-Gruppen oder ähnliche digitale Communitys haben sich in Krisensituationen wie Naturkatastrophen oder terroristischen Anschlägen als wichtige Mittel zur schnellen Verbreitung von Informationen herausgestellt [30]. Allerdings können besonders in diesem Feld ein fehlendes oder ein zu großes Vertrauen in Informationen [31], das Verbreiten von irrelevanten und Fehlinformationen sowie die Entstehung von auf solchen Fehlinformationen beruhenden ungünstigen Verhaltensweisen zu erheblichen Problemen [32] führen.

\section{Krankheitsprävention}

Im Bereich der Prävention von Erkrankungen, vor allem der Prävention von lebensstilbezogenen Erkrankungen, lassen sich mehrere Ansatzpunkte für digitale Technologien identifizieren, angefangen von der personalisierten Identifikation von Risikofaktoren für Krankheiten und daraus abgeleiteten personalisierten Empfehlungen zur Verhaltensänderung oder Inanspruchnahme präventiver medizinischer Leistungen (z. B. Onlinetools zur Berechnung des familiären Risikos für Krankheiten mit genetischem Risiko; [33]) bis hin zu mobilen Anwendungen (Apps) zur Unterstützung oder direkten Modifikation von individuellen Verhaltensweisen. Insbesondere in diesem Bereich bündeln sich kommerzielle Interessen, was auch darin begründet ist, dass sich Lebensstil-Apps (relativ) schnell und unkompliziert programmieren lassen, insbesondere wenn die Entwicklung nicht nach evidenzbasierten Prinzipien erfolgt (z. B. [34, 35]). Dadurch kommt es zu einer unübersehbaren Vielzahl von Angeboten auf dem Markt (aktuell knapp 100.000 Apps in der Health-and-FitnessKategorie sowie weitere knapp 45.000 Appsin der Medical-Kategorie im Google Play Store (Google Ireland Ltd, Dublin, Irland); [36]), von denen allerdings nur ein verschwindend geringer Anteil reguliert oder evidenzbasiert entwickelt wurde.

\section{Gesundheitsförderung}

Auch in diesem Bereich liegt eine Vielzahl von online- und mobilbasierten Angeboten vor, die denselben Herausforderungen und Schwierigkeiten wie Apps im Bereich der Prävention von Erkrankungen unterliegen - vielleicht sogar noch stärker, weil sowohl Risikofaktoren als auch Ressourcen für Gesundheit unspezifischer und daher möglicherweise schwerer zu verändern sind als im Bereich der Prävention spezifischer Erkrankungen. Wir möchten hier insbesondere auf die Identifikati- on von Determinanten und Techniken zur Verhaltensänderung in mobilen Anwendungen aufmerksam machen (für Apps, die zur Reduktion von sedentärem Verhalten beitragen sollen, siehe z.B. [37]) und auf die Tatsache, dass die große Mehrzahl von Anwendungen solche Determinanten gar nicht erst verwendet oder adressiert. Auch hier stellt die Entwicklung von Anwendungen, die die Bedarfe und Präferenzen von spezifischen Adressatengruppen berücksichtigen, eine große Herausforderung dar (z. B. [38]).

Diese subjektive und selektive Übersicht von digitalen Gesundheitstechnologien bestätigt, dass in den Kernfunktionen von Public Health digitale Technologien eingesetzt werden können und dies aktuell auch schon stattfindet. Gleichzeitig zeigt dieser Überblick aber auch, dass ohne eine Einordnung digitaler Technologien in ein übergeordnetes Rahmenkonzept von Public Health und ohne eine solide Evidenzbasis der technologische Fortschritt und nicht evidenzbasierte und bedarfsgerechte Public-HealthÜberlegungen die Entwicklung vorantreiben werden. Im Folgenden illustrieren wir dieses Spannungsfeld am Beispiel der digitalen Spaltung.

\section{Das Problem der digitalen Spaltung}

Ein großes Problem von Digital Public Health ist der „schmale[n] Grat zwischen Autonomie und externer Kontrolle bei der automatisierten Erfassung von Gesundheitsdaten", wie es treffend von Dockweiler und Razum [39] formuliert wurde. Zwar erlaubt die Erfassung großer Datenmengen eine bessere Prozessevaluation und somit auch eine Optimierung von Public-Health-Programmen, aber sie birgt auch die Gefahr eines kritischen Ungleichgewichts zwischen den Entwicklern von Gesundheitstechnologien auf der einen und Nutzern sowie Nutzerinnen auf der anderen Seite. Entwickler von Gesundheitstechnologien können Daten aus dem Gebrauch ihrer Produkte ziehen und teilweise kommerziell weiterverwerten, während die Nutzer, die die relevanten und persönlichen Gesundheitsdaten produzieren, nicht ohne Weiteres 
auf die Daten zugreifen können, mithin auch ihre Nutzungsrechte abgeben [6]. Dieses Problem wird dadurch verstärkt, dass zum einen die sogenannte Digital Health Literacy, d.h. die Kompetenz im Umgang mit digitalen Technologien im Gesundheitsbereich, in der Gesellschaft ungleich verteilt ist [40]. Zum anderen ist der Zugang zu digitalen Technologien nicht in allen Bevölkerungsgruppen uneingeschränkt möglich, sei dies aus versorgungstechnischen Gründen (z.B. erschwerter Zugang zu Breitbandinternet im ländlichen Raum), aus Gründen mangelhafter Barrierefreiheit von digitalen Angeboten oder aus Gründen mangelhaft entwickelter Technologie, die bestimmte Adressaten nicht repräsentiert oder nicht gezielt anspricht [14]. Digitale Ungleichheit, d. h. eine ungleiche Verfügbarkeit digitaler Technologien in der Gesellschaft, kann jedoch u. a. mit einem ungleichen Zugang zu Informationen über Gesundheitsversorgung, ungleichen $\mathrm{Zu}$ gängen $\mathrm{zu}$ Therapien und ungleichem Zugang zu Ressourcen für Gesundheitsförderung und Prävention einhergehen. Dies kann einerseits zu einer Vergrößerung von sozioökonomischer und gesundheitlicher Ungleichheit führen. Andererseits lassen sich Ansätze identifizieren, wie durch eine Verringerung der digitalen Spaltung und der ausgewogenen Nutzung neuer Technologien Ungleichheit bzw. gesundheitliche Ungerechtigkeiten vermindert werden können $[4,41$, 42].

Im vorliegenden Heft beschäftigen sich die Beiträge von Cornejo-Müller et al. [43] sowie Schüz und Urban [44] ausführlicher mit Problemen und Risiken, die sich aus der ungleichen Verteilung von digitalen Ressourcen und der ungleich verteilten Nutzung von digitalen Angeboten ergeben können.

\section{Datenschutz im Zeichen von Digital Public Health}

Mit dem Sammeln von großen Datenmengen und ihrer Analyse anhand häufig undurchsichtiger Algorithmen kann sowohl Nutzen als auch Risiko verbunden sein. So können wir z.B. ein Interventionsprogramm entwickeln, das besser auf ein bestimmtes Indivi- duum abgestimmt ist, und in Echtzeit seine Nutzung durch die jeweilige Person optimieren (sogenannte Just-inTime Adaptive Interventions [JITAIs] [19]), aber wie garantieren wir, dass die erhobenen Daten nicht auch für andere Zwecke verwendet werden, die z.B. nicht durch die gegebene Einverständniserklärung abgedeckt sind? In Zeiten des Anspruchs, einmal erhobene Forschungsdaten auch anderen Forschern zur Verfügung zu stellen (Data Sharing), indem ein Forschungsdatenmanagement unter Berücksichtigung der $\operatorname{FAIR}(\mathrm{F}=$ Findable, $\mathrm{A}=$ Accessible, $\mathrm{I}=$ Interoperable,$\quad \mathrm{R}=$ Reusable)-Data Prinzipien aufgesetzt wird, wird Digital Public Health vor große Herausforderungen gestellt.

Grundsätzlich handelt es sich bei Gesundheitsdaten um sensible Daten, die eines besonderen Schutzes bedürfen. Ein solcher Schutz ist aber schwer sicherzustellen, wenn z.B. mithilfe von Gesundheitstrackern Informationen von Personen erhoben werden, die direkt von großen Internetkonzernen weiterverarbeitet werden können - zumeist ohne weitere Möglichkeit zur Einsicht durch Einzelpersonen. Ein möglicher Weg, der zumindest im Rahmen konkreter Forschungsvorhaben erhobene sensible Daten vor Missbrauch schützen kann, sieht distribuierte Datenanalysen vor, bei denen die Daten im jeweiligen Forschungsinstitut verbleiben und spezielle Analyseprogramme eingesetzt werden (z.B. DataSHIELD [45]), die keine Zusammenführung der Rohdaten erfordern. Eine andere Möglichkeit besteht darin, einen Vertrag mit möglichen Nutzern abzuschließen, nachdem geprüft wurde, ob der angestrebte Forschungszweck durch die gegebene Einverständniserklärung abgeklärt ist, und diesen anschließend einen auf ihre Forschungszwecke abgestimmten Analysedatensatz zur Verfügung zu stellen. In diesem Zusammenhang sind auch Ansätze wie der Personal Health Train [46] zu erwähnen. Dabei handelt es sich um eine verteilte Infrastruktur, die die (Nach-)Nutzung von Gesundheitsdaten ermöglichen soll und die das Datenmanagement, die Datenana- lyse und medizinische Entscheidungen unterstützt.

\section{Diskussion}

Das Potenzial digitaler Technologien im Bereich Public Health liegt in der Möglichkeit, (1) eine große Anzahl von Personen zu vergleichsweise geringen Kosten zu erreichen, (2) den direkten Kontakt (und die damit verbundenen Kosten) zu Personen bei der Implementation von Public-Health-Programmen zu reduzieren, (3) verschiedene Dimensionen effektiver Public-Health-Interventionen parallel zu adressieren und (4) große Datenmengen und objektive Messungen zu generieren, die wiederum für die Evaluation, das Monitoring und schlussendlich die Optimierung von Public-Health-Programmen genutzt werden können. Darüber hinaus kann die Bildung digitaler Gemeinschaften - sei es im Rahmen von spezifischen Programmen oder allgemeiner durch soziale Medien - dazu beitragen, ein gesundheitsförderndes Umfeld zu schaffen.

Allerdings sind mit Digital Public Health auch zahlreiche ungelöste und potenziell kritische Aspekte verbunden. Diese betreffen insbesondere die Sorge um den Schutz der Privatsphäre, Eigentumsrechte an den Gesundheitsdaten, die durch die Benutzung von Technologie entstanden sind, und das insgesamt doch starke Vertrauen auf technologische Entwicklungen statt auf persönliche Beziehungen und Engagement für und in der Gemeinschaft. Dazu kommen die vielen Stimmen, die davor warnen, dass z. B. durch die unterschiedlich ausgeprägte Kompetenz im Umgang mit digitalen Technologien sozioökonomische und damit auch gesundheitliche Ungleichheiten eher verstärkt werden.

In Bezug auf die Evaluation digitaler Public-Health-Ansätze ist derzeit noch von erheblichen Beschränkungen auszugehen. Malvey und Slovensky [47] zählen das Fehlen angemessener Evaluationsmodelle $\mathrm{zu}$ den wesentlichen Limitationen bei der Förderung von M-Health aus globaler Sicht und konstatieren, dass die Entwicklung von Evaluationsmethoden nicht mit der hohen Geschwindigkeit der technologischen 
Entwicklung Schritt gehalten hat. Ein zentraler Ansatz sind derzeit systematische Literaturübersichten von E- und M-Health-Interventionen. Dieser Ansatz ist im Sinne der Anforderungen an eine Evidenzbasierung zu begrüßen, allerdings stellt sich auch hier die Frage, ob die Methodik angesichts der schnellen Technologieentwicklung adaptiert und ergänzt werden muss. Ein Gegenargument kann darin begründet liegen, dass sich nur überdauernd und mehrfach eingesetzte E- und M-Health-Maßnahmen in Public Health durchsetzen können, die dann wiederum Evidenzsynthesen zugänglich sind. Allerdings sind auch bei diesen Maßnahmen die für die Evaluation nutzbaren Follow-up-Zeiträume oft sehr eingeschränkt, sodass überdauernde Wirkungen (erwünscht wie unerwünscht) kaum zu beurteilen sind. Insgesamt ist von erheblichem Diskussions- und Entwicklungsbedarf in Bezug auf die Evaluation von Digital Public Health auszugehen.

\section{Fazit}

Auch wenn Digital Public Health grundsätzlich neue Wege erlaubt, um mit individuell zugeschnittenen Präventions- und Gesundheitsförderungsprogrammen breite Bevölkerungsgruppen anzusprechen, sind umfassende Evaluationen von Digital-Public-HealthProgrammen immer noch selten und angemessene Evaluationsparadigmen stehen aus. Aspekte wie Nachhaltigkeit von kurzfristigen Effekten und adäquate Berücksichtigung der Nutzerperspektive wurden zudem bislang nicht ausreichend thematisiert.

\section{Korrespondenzadresse}

\section{Prof. Dr. Hajo Zeeb}

Leibniz-Institut für Präventionsforschung und Epidemiologie - BIPS

Achterstr. 30, 28359 Bremen, Deutschland zeeb@leibniz-bips.de

Danksagung. Wir bedanken uns für die Unterstützung durch den Leibniz-WissenschaftsCampus Digital Public Health Bremen (Isc-diph.de), der gemeinsam von der Leibniz-Gemeinschaft (W4/2018), der Freien Hansestadt Bremen und dem LeibnizInstitut für Präventionsforschung und Epidemiologie - BIPS gefördert wird.

\section{Einhaltung ethischer Richtlinien}

Interessenkonflikt. H. Zeeb, I. Pigeot und B. Schüz geben an, dass kein Interessenkonflikt besteht.

Für diesen Beitrag wurden von den Autoren keine Studien an Menschen oder Tieren durchgeführt. Für die aufgeführten Studien gelten die jeweils dort angegebenen ethischen Richtlinien.

Open Access. Dieser Artikel wird unter der Creative Commons Namensnennung 4.0 International Lizenz veröffentlicht, welche die Nutzung, Vervielfältigung, Bearbeitung, Verbreitung und Wiedergabe in jeglichem Medium und Format erlaubt, sofern Sie den/die ursprünglichen Autor(en) und die Quelle ordnungsgemäß nennen, einen Link zur Creative Commons Lizenz beifügen und angeben, ob Änderungen vorgenommen wurden.

Die in diesem Artikel enthaltenen Bilder und sonstiges Drittmaterial unterliegen ebenfalls der genannten Creative Commons Lizenz, sofern sich aus der Abbildungslegende nichts anderes ergibt. Sofern das betreffende Material nicht unter der genannten Creative Commons Lizenz steht und die betreffende Handlung nicht nach gesetzlichen Vorschriften erlaubt ist, ist für die oben aufgeführten Weiterverwendungen des Materials die Einwilligung des jeweiligen Rechteinhabers einzuholen.

Weitere Details zur Lizenz entnehmen Sie bitte der Lizenzinformation auf http://creativecommons.org/ licenses/by/4.0/deed.de.

\section{Literatur}

1. Müller AM, Maher CA, Vandelanotte $C$ et al (2018) Physical activity, sedentary behavior, and diet-related eHealth and mHealth research: bibliometricanalysis.JMed InternetRes 20(4):e122

2. Nyarku M, Mazaheri M, Jayaratne R et al (2018) Mobile phones as monitors of personal exposure to air pollution: is this the future? PLOS ONE 13:e193150

3. Brabham DC, Ribisl KM, Kirchner TR, Bernhardt JM (2014) Crowdsourcing applications for public health. Am JPrev Med 46(2):179-187

4. Chou WYS, Prestin A, Lyons C, Wen KY (2013) Web 2.0 for health promotion: reviewing the current evidence. Am J Public Health 103(1):e9-e18

5. Chauvin J, Lomazzi M(2017) The digital technology revolution and its impact on the public's health. Eur JPublic Health 27(6):947

6. Lupton D (2018) Digital health: critical and crossdisciplinary perspectives. Routledge, London

7. Nievas Soriano BJ, García Duarte S, Fernández Alonso AM, Bonillo Perales A, Parrón Carreño T (2019) ehealth: advantages, disadvantages and guiding principles for the future. JMIR. https://doi. org/10.2196/preprints.15366

8. Oh H, Rizo C, Enkin M, Jadad A (2005) What is eHealth?: a systematic review of published definitions. World Hosp Health Serv 41:32-40

9. Shaw T, McGregor D, Brunner M, Keep M, Janssen A, Barnet S (2017) What is eHealth (6)? Development of a conceptual model for eHealth: qualitative study with key informants. J Med Internet Res 19:e324

10. Public Health England (2017) Digital-first public health: public health England's digital strategy. https://www.gov.uk/government/publications/ digital-first-public-health/digital-first-public- health-public-health-englands-digital-strategy. Zugegriffen: 1.Aug. 2019

11. Davis $T L$, Diclemente $R$, Prietula M (2016) Taking mHealth forward: examining the core characteristics. JMIRMhealth Uhealth 4:e97

12. Knöppler K, Neisecke T, Nölke L (2016) DigitalHealth-Anwendungen für Bürger: Kontext, Typologie und Relevanz aus Public-Health-Perspektive; Entwicklung und Erprobung eines Klassifikationsverfahrens. Bertelsmann, Gütersloh

13. Oldenburg B, Taylor CB, O'Neil A, Cocker F, Cameron LD (2015) Using new technologies to improve the prevention and management of chronic conditions in populations. Annu Rev Public Health 36:483-505

14. Veinot TC, Mitchell H, Ancker JS (2018) Good intentions are not enough: how informatics interventions can worsen inequality. J Am Med Inform Assoc 25:1080-1088

15. Dadaczynski K, Tolks D (2018) Digitale Public Health:Chancen und Herausforderungen internetbasierter Technologien und Anwendungen. Public Health Forum 26(3):275-278

16. Brownson RC, Fielding JE, Green LW (2018) Building capacity for evidence-based public health: reconciling the pulls of practice and the push of research. Annu Rev Public Health 39:27-53

17. Murray E, Hekler EB, Andersson G et al (2016) Evaluating digital health interventions: key questions and approaches. Am J Prev Med 51:843-851

18. Roess A (2017) The promise, growth, and reality of mobile health - another data-free zone. N Engl J Med 377:2010-2011

19. Hardeman W, Houghton J, Lane K, Jones A, Naughton F (2019) A systematic review of just-intime adaptive interventions (JITAIs) to promote physical activity. Int JBehav Nutr Phys Act 16:31

20. National Institute for Health and Care Excellence (2019) Evidence standards framework for digital health technologies. https://www.nice. org.uk/about/what-we-do/our-programmes/ evidence-standards-framework-for-digitalhealth-technologies. Zugegriffen:2. Aug. 2019

21. World Health Organization (2012) The new European policy for health-Health 2020. Policy framework and strategy. Draft 2. https:// www.who.int/workforcealliance/knowledge/ resources/Health2020_long.pdf. Zugegriffen: 8. Aug. 2019

22. Nationale Akademie der Wissenschaften Leopoldina, Acatech (2015) Public Health in Deutschland - Strukturen, Entwicklungen und globale Herausforderungen. Leopoldina, Halle

23. Brockmann D (2020) Digitale Epidemiologie. Bundesgesundheitsblatt Gesundheitsforschung Gesundheitsschutz. https://doi.org/10.1007/ s00103-019-03080-z

24. Geneviève LD, Martani A, Wangmo T et al (2019) Participatory disease surveillance systems: ethical framework. J Med Internet Res 21:e12273

25. Geneviève LD, Wangmo T, Dietrich D, WoolleyMeza O, Flahault A, Elger BS (2018) Research ethics in the European Influenzanet Consortium: scoping review. JMIR Public Health Surveill 4:e67

26. Ginsberg J, Mohebbi MH, Patel RS, Brammer L, Smolinski MS, Brilliant L (2008) Detecting influenza epidemics using search engine query data. Nature 457:1012

27. Butler D (2013) When Google got flu wrong. Nature 494:155-156

28. Merchant RM, Elmer S, Lurie N (2011) Integrating social media into emergency-preparedness efforts. N Engl J Med 365:289-291 


\section{Leitthema}

29. Markenson D, Howe L (2014) American Red Cross Digital Operations Center (DigiDOC): an essential emergency management tool for the digital age. Disaster Med Public Health Prep 8:445-451

30. Freeman JD, Blacker B, Hatt G et al (2019) Use of big data and information and communications technology in disasters: an integrative review. Disaster Med Public Health Prep 13:353-367

31. Lachlan KA, Spence PR, Edwards A, Reno KM, Edwards C (2014) If you are quick enough, I will think about it: information speed and trust in public health organizations. Comput Human Behav 33:377-380

32. Eckert S, Sopory P, Day A et al (2018) Health-related disaster communication and social media: mixedmethod systematic review. Health Commun 33(12):1389-1400

33. Welch BM, Wiley K, Pflieger L et al (2018) Review and comparison of electronic patient-facing family health history tools. J Genet Couns 27:381-391

34. Albrecht UV, von Jan U (2017) Safe, sound and desirable: development of mHealth apps under the stress of rapid life cycles. mHealth 3:27. https:// doi.org/10.21037/mhealth.2017.06.05

35. van Heerden A, Tomlinson M, Swartz L (2012) Point of care in your pocket: a research agenda for the field of m-health. Bull World Health Organ 90:393-394. https://doi.org/10.2471/BLT. 11.099788

36. 42 matters (2019) Google Play ${ }^{\mathrm{TM}}$ store stats vs. iOS ${ }^{\circledR}$ App Store ${ }^{\circledR}$ stats. https://42matters.com/stats. Zugegriffen: 1. Aug. 2019

37. Dunn EE, Gainforth $\mathrm{HL}$, Robertson-Wilson JE (2018) Behavior change techniques in mobile applications for sedentary behavior. Digit Health. https://doi.org/10.1177/2055207618785798

38. Wichmann F, Sill J, Hassenstein MJ, Zeeb H, Pischke CR (2019) Apps zur Förderung von körperlicher Aktivität.Präv Gesundheitsf 14:93-101

39. Dockweiler C, Razum O (2016) Digitalisierte Gesundheit: neue Herausforderungen für Public Health. Gesundheitswesen 78(01):5-7

40. Samerski S, Müller H (2019) Digitale Gesundheitskompetenz in Deutschland - gefordert, aber nicht gefördert? Ergebnisse der empirischen Studie TKDiSK. Z Evid Fortbild Qual Gesundhwes. https:// doi.org/10.1016/j.zefq.2019.05.006

41. Graham GN, Ostrowski M, Sabina AB (2016) Population health-based approaches to utilizing digital technology: a strategy for equity. J Public Health Policy 37(2):154-166

42. Latulippe K, Hamel C, Giroux D (2017) Social health inequalities and eHealth: a literature review with qualitative synthesis of theoretical and empirical studies. J Med Internet Res 19:e136

43. Cornejo-Müller A, Wachtler B, Lampert T (2020) Digital Divide - soziale Unterschiede in der Nutzung digitaler Gesundheitsangebote. Bundesgesundheitsblatt Gesundheitsforschung Gesundheitsschutz. https://doi.org/10.1007/s00103-01903081-y

44. Schüz B, Urban M (2020) Unerwünschte Effekte digitaler Gesundheitstechnologie: eine PublicHealth-Perspektive. Bundesgesundheitsblatt Gesundheitsforschung Gesundheitsschutz. https:// doi.org/10.1007/s00103-019-03088-5

45. DataSHIELD (2019) DataSHIELD—a software solution for secure bioscience collaboration. http://www.datashield.ac.uk/.Zugegriffen:7.Aug. 2019

46. Dutch Techcentre for Life Sciences (2019) Personal health train. https://www.dtls.nl/fair-data/ personal-health-train/.Zugegriffen: 7. Aug. 2019
47. Malvey DM, Slovensky DJ (2017) Global mHealth policy arena: status check and future directions. mHealth 3:41. https://doi.org/10.21037/mhealth. 2017.09.03 